\title{
Perspectivas Comparadas entre los Docentes de Posgrado de Investigadores en Educación de la UNAM y la UCM
}

\author{
Compared Perspectives among Faculty Members of \\ Postgraduate School at UNAM and UCM
}

\author{
María R. Belando-Montoro ${ }^{1 *}$ \\ José Francisco Alanís Jiménez ${ }^{2}$ \\ ${ }^{1}$ Universidad Complutense de Madrid, España \\ ${ }^{2}$ Universidad Nacional Autónoma de México, México
}

\begin{abstract}
El propósito de este estudio es indagar las principales características del buen docente universitario de posgrados de investigadores en educación para poder construir una perspectiva desde la que analizar la calidad de la formación que imparten. El aporte original frente a otros estudios que han tratado cuestiones similares es que, en este caso, la muestra utilizada en la investigación está constituida por docentes de los posgrados de dos de las universidades más importantes de Iberoamérica. La metodología se basó en una estrategia de estudio de casos múltiples, para lo cual se utilizó un cuestionario de preguntas abiertas que se pasó a docentes de dichos posgrados. Los resultados muestran el predominio de la competencia cognitiva en la caracterización del buen docente, así como en las ideas sobre cómo llegar a serlo, mientras que en sus recomendaciones para mejorar el trabajo académico se subrayó la importancia de la competencia social. En las conclusiones se cuestiona el hecho de que en el posgrado se esté dando tanta importancia a la Competencia cognitiva, cuando a nivel mundial se está pidiendo replantear la educación considerando criterios humanísticos sensibles a las necesidades sociales, más afines con la Competencia ética y la Competencia social.
\end{abstract}

Descriptores: Profesor de enseñanza superior; Posgrado; Calidad de la educación; México; España.

This study has the purpose of researching the main characteristics of the good university professor of postgraduates for educational researchers in order to build a perspective of the quality of the training they teach. The main difference with similar researches is that, in this case, the study used a pool of graduate school teachers from two of the most important universities of Latin America and Spain. The methodology for this research was based on a multiple case study strategy, with a questionnaire designed for this purpose, which was given to faculty members of graduate school at the UNAM and the UCM. Results show a high incidence in cognitive competence as a key feature to recognize a good teacher, as well as for how to become one of them. Faculty members also recommended focusing on social competence when trying to improve academic work. The conclusions question the fact that the postgraduate level is giving so much importance to Cognitive competence, when at a global level, it is being asked to rethink education considering humanistic criteria sensitive to social needs, more akin to Ethical competence and Social competence.

Keywords: University professors; Postgraduate courses; Educational quality; Mexico; Spain.

*Contacto: mbelando@edu.ucm.es

ISSN: $1696-4713$

www.rinace.net/reice/

revistas.uam.es/reice
Recibido: $\quad 14$ de enero 2019

$1^{\text {a }}$ Evaluación: 28 de febrero 2019

$2^{\text {a }}$ Evaluación: 10 de marzo 2019

Aceptado: 1 de mayo 2019 


\section{Introducción}

En los últimos años, tanto en el ámbito europeo como en el latinoamericano, las universidades han experimentado cambios importantes debido a las nuevas políticas supranacionales. El denominado Proceso de Bolonia ha modificado las estructuras de la Educación Superior en todo el espacio europeo pero las prioridades políticas han ido cambiando a lo largo del tiempo y se han ido centrando en aspectos esenciales como la calidad. Para garantizar y mejorar la calidad se han creado, o convertido, organismos específicos que tienen, entre otros objetivos, la evaluación del profesorado. En el caso de España, la Agencia Nacional de Evaluación de la Calidad y Acreditación (ANECA), creada en 2002, y que a partir de 2014 concentró también las competencias de la Comisión Nacional Evaluadora de la Actividad Investigadora (CNEAI), es responsable, entre otros, de las funciones de evaluación y acreditación del profesorado universitario. Dichas funciones también son asumidas por agencias creadas en diversas comunidades autónomas.

En el marco de dicho propósito de mejora de la calidad, el grupo de alto nivel para la modernización de la enseñanza superior de la Unión Europea publicó, en junio de 2013, un informe sobre la mejora de la calidad de la enseñanza y el aprendizaje en las universidades (McAleese et al., 2013) en el que se hicieron dieciséis recomendaciones entre las que se incluyen varias concretas sobre el profesorado, destacando su formación pedagógica, su formación permanente y la evaluación de las competencias docentes. También en el ámbito iberoamericano se ha prestado atención a la necesidad de la mejora de sus instituciones de educación superior y a sus niveles de calidad, especialmente desde mediados de los 90 (Fernández, 2007), fruto de ello es la creación de organismos y asociaciones tales como la Red Iberoamericana para el Aseguramiento de la Calidad en la Educación Superior (RIACES) en 2003.

En México, el escenario para la evaluación de la educación superior y sus docentes está de alguna manera más revuelto, siendo que la sistematización de los esfuerzos en este sentido se conforma por una amalgama de instancias públicas y de la sociedad civil. Así, esta función que inicialmente ejerció la ANUIES (Asociación Nacional de Universidades e Instituciones de Educación Superior, una Asociación Civil), se apoyó en organismos gubernamentales regionales denominados COEPES (Comités Estatales para la Planeación de la Educación Superior) dependientes de la Secretaría de Educación Pública (SEP). Posteriormente, la capacidad de certificar la calidad de la educación superior se confirió a los CIEES (Comités Interinstitucionales para la Evaluación de la Educación Superior), a los cuales se apoyó con la creación del COPAEs (Consejo para la Acreditación de la Educación Superior) con la finalidad de regular las instancias acreditadoras de instituciones y programas de educación superior con mayor homogeneidad en sus criterios de calidad, sin embargo la ANUIES no dejó de tener influencia al interior de este nuevo organismo. Por su parte el CONACYT (Consejo Nacional de Ciencia y Tecnología, organismo descentralizado del gobierno federal), acredita programas de posgrado incorporándolos a su Programa Nacional de Posgrados de Calidad (PNPC), y paralelamente, la FIMPES (Federación de Instituciones Mexicanas Particulares de Educación Superior), desarrolló su propio sistema de certificación para sus miembros en el sector privado.

En cuanto a la certificación de académicos en lo individual, el PRODEP (Programa para el Desarrollo Profesional Docente, para el Tipo Superior, que depende de la SEP) establece 
los criterios del "perfil deseable" para profesores de tiempo completo en instituciones públicas, combinando dentro de éste actividades de docencia, investigación, gestión y extensión. También el CONACYT otorga una distinción y estímulos a investigadores a través del SNI (Sistema Nacional de Investigadores) incluyendo la docencia de nivel superior como uno de sus requisitos, pero sin evaluar su calidad.

Aparte de todo lo mencionado en los párrafos precedentes, la Universidad Nacional Autónoma de México (UNAM) cuenta con sus propios sistemas de reconocimientos y estímulos a docentes y académicos, que dependen de la Dirección General de Asuntos para el Personal Académico (DGAPA), en tanto que se encarga a cada Facultad la evaluación de su profesorado contando con la asesoría y apoyo de la Dirección General de Evaluación Educativa, la cual a su vez cuenta con diversos programas para ello.

Pero a pesar de todo este aparato burocrático, es posible que los mayores niveles de reconocimiento y estímulos sobre las trayectorias académicas no necesariamente coincidan con un mejor ejercicio de la docencia (Hortigüela et al., 2017; Matus y Linares, 2016; Rocha, 2012), en tanto que las evaluaciones basadas en las opiniones del alumnado también han sido cuestionadas por no reflejar adecuadamente la calidad del profesorado (Arbesú et al., 2011; Moreno, 2018; Reyero, 2014).

A pesar de los esfuerzos realizados en estos últimos años y los avances significativos, todavía hoy los retos sobre la calidad en las instituciones de educación superior son numerosos. En este estudio nos centraremos en dos problemas detectados en el "Informe sobre la educación superior en América Latina y el Caribe 2000-2005", publicado por el Instituto Internacional de la UNESCO para la Educación Superior en América Latina y el Caribe (IESALC), relacionados con los estudios de posgrado y la formación del profesorado.

Si bien durante la década del 90 se registró en toda América Latina -con mayor énfasis en algunos países como Argentina, Chile, Colombia, México y Brasil- el desarrollo de diversos posgrados, los cuerpos académicos consolidados con esa formación son aún pequeños y su distribución en las instituciones de educación superior es insuficiente y desigual. A esto hay que agregarle la deficiencia en los sistemas de selección, desarrollo y promoción del personal de las instituciones de educación superior y la falta de incentivos para elevar los niveles de formación (Fernández, 2007, p. 36).

En línea con lo argumentado, en este trabajo pretendemos ofrecer una comparación de perfiles del buen profesorado universitario de posgrado de investigadores en educación, esbozar los procesos formativos de los docentes en este nivel y hacer recomendaciones para la mejora del trabajo académico. Todo ello a partir de la voz de quienes bien o mal, se encuentran realizando esta tarea en forma cotidiana, responsables del desarrollo de los más altos niveles de profesionalización, en dos de las instituciones universitarias más importantes de Iberoamérica: la Universidad Nacional Autónoma de México (UNAM) y la Universidad Complutense de Madrid (UCM). Para abordar estas cuestiones, comenzaremos revisando la literatura iberoamericana (mayormente de México y España ya que son los contextos en los que se centra este estudio) más relevante en este ámbito, de modo que podamos delimitar elementos que nos permitan más adelante enfocar la naturaleza y contenido de la buena docencia y la calidad en los posgrados en dicho entorno. 


\section{Sobre la buena docencia universitaria y la atención a la investigación}

En España la atención a la calidad y la excelencia docente se ha visto impulsada especialmente a partir del inicio del proceso de convergencia hacia un Espacio Europeo de Educación Superior, de aquí que a partir del año 2000 se hayan visto multiplicadas las publicaciones al respecto, especialmente sobre la cuestión más amplia de calidad y del buen docente universitario (Casero, 2010; Fernández-Cruz y Romero, 2010; García-Gallego et al., 2015; Gargallo et al., 2010; Hamer, 2015; Miguel, 2003; Monereo y Domínguez, 2014; Reyero, 2014; Villa, 2008). En México la literatura acerca de la buena docencia, la docencia de calidad o las buenas prácticas del docente universitario es también vasta (por ejemplo, Carlos, 2011; García y Medécigo, 2014; Luna, Valle y Osuna, 2010; Patiño, 2015; Rocha, 2012, 2013).

La importancia de la formación inicial y continua del profesor universitario ha sido subrayada por numerosos autores (Bausela, 2004; Fernández, 2008; Gargallo et al., 2010) así como, específicamente, la relevancia de la formación pedagógica del profesorado y su relación con la calidad de la enseñanza universitaria (Miguel, 2003).

Una docencia universitaria de calidad teóricamente se identifica con un amplio listado de competencias (Ruiz-Corbella y Aguilar-Feijoo, 2017; Torra y otros, 2012), mientras que en la práctica esto no se está valorando como parte de una formación necesaria para el profesorado universitario (Zabalza 2009). Así, en España el profesorado de posgrado en general está principalmente orientado a la investigación, lo que también ocurre en el caso particular de la UNAM, por lo que las habilidades para la buena docencia suelen ser aprendidas a través de la propia experiencia y relaciones con otros docentes.

La divergencia entre la teoría y la práctica del buen desempeño de la docencia universitaria se señala igualmente en una investigación de Monereo y Domínguez (2014), en la que se confrontan competencias identificadas por ocho universidades catalanas frente a las percepciones de 20 profesores sobresalientes. Las conclusiones revelaron que sí hay un modelo de perfil de competencias, pero hay cinco aspectos en los que se mostraban discrepancias (Monereo y Domínguez, 2014).

Desde luego, los resultados de las investigaciones pueden variar dependiendo de quiénes sean los actores que participan en ellas, así como la fuente de donde surgen los parámetros de excelencia y buenas prácticas. Los estudios sobre la buena docencia universitaria se han desarrollado teniendo en cuenta el punto de vista del alumnado, del profesorado o de ambos, mostrando numerosas coincidencias entre sus percepciones (Belando-Montoro y Tavárez, 2017; Casero; 2010; Gargallo et al., 2010).

Sin embargo, las acreditaciones y evaluaciones formales del docente de posgrado casi siempre parten de parámetros institucionales en los que los criterios para definir la excelencia o caracterizar la buena docencia suelen estar más relacionados con los grados académicos, las publicaciones indexadas y las investigaciones financiadas, entre otros, lo que en ocasiones lleva a comportamientos que podrían calificarse de extravagantes, como algunos de los descritos por Vera (2017) al referirse a la evaluación cuantitativa del mérito 
académico que se lleva a cabo en una institución de educación superior pero que es cada vez más habitual tanto en el ámbito universitario latinoamericano como en el europeo. ${ }^{1}$

Partiendo desde la perspectiva de docentes de excelencia, Fernández-Cruz y Romero (2010) obtuvieron diez dimensiones y con ellas construyeron un cuestionario que remitieron a todo el profesorado de la Universidad de Granada. Entre los resultados obtenidos sobre los indicadores que mejor definen la excelencia docente, destacamos los siguientes: el profesorado conoce profundamente la materia que imparte; promueve la implicación de sus estudiantes en el aprendizaje, prepara bien sus clases y se preocupa de motivar a sus estudiantes (Fernández-Cruz y Romero, 2010, p. 91-114).

Por su parte, desde México, la docencia efectiva unida a la educación humanista fue el objeto de estudio de la investigación de Patiño (2012). Entre las principales conclusiones obtenidas destaca el interés que los docentes efectivos tienen hacia el aprendizaje del alumno y su no creencia hacia el aprendizaje memorístico, dan valor al diálogo, y en este a la escucha activa, entre otras. También en este país, Guzmán (2018) entrevistó a profesores seleccionados por su calidad docente y los resultados mostraron su dominio tanto de los aspectos didácticos como de los contenidos de sus disciplinas, su compromiso y disfrute en la tarea de enseñar y su interés por tener buenas relaciones con sus estudiantes.

Atendiendo a la literatura analizada, el perfil del buen profesor podría caracterizarse a través de los siguientes descriptores: dominio disciplinar, formación pedagógica continua, manejo de tecnologías de la información y comunicación (TIC), competencias diversas vinculadas a planes formativos, innovación, respeto al estudiante, investigación, evaluación con justicia, ser colaborativo, practicar la docencia relacional centrada en el estudiante y su aprendizaje, promover el pensamiento crítico y reflexivo, motivar al estudiante para que construya conocimiento y aprenda por sí mismo, ser dialógico, desconfianza en la memorización, escucha activa y generación de un clima de confianza. La relación es tan extensa, y no exenta de contradicciones, que surge la interrogante acerca de la posibilidad de que estas variaciones y diversidades obedezcan a circunstancias contextuales, y si sería posible que existieran coincidencias entre las percepciones del profesorado de posgrado de dos grandes universidades iberoamericanas de distintos continentes. Hacemos notar en este punto que la literatura sobre casos con estas características específicas es inexistente.

\section{Método}

Martínez (2010) destaca la conveniencia de que participen diferentes actores en la definición de criterios de profesionalización de los docentes, sin embargo, consideramos igualmente que le corresponde a cada grupo específico de profesionales, y en este caso a los docentes, la voz principal; la Prima Voce para determinar las características de las buenas prácticas dentro de su profesión. Por ello esta investigación se centró en caracterizar al buen profesor de posgrado de investigadores en educación, desde su propia perspectiva.

\footnotetext{
${ }^{1}$ Uno de los ejemplos típicos es la conducta de un docente que, teniendo material para escribir un libro, decide escribir en su lugar cuatro artículos ya que institucionalmente tiene más valor.
} 
Con esta elección en mente, la metodología que se siguió para esta investigación se basó en la estrategia de estudio de casos múltiples (Yin, 1989). De acuerdo con esta, la investigación se diseña siguiendo una lógica de réplica, y no de muestreo. Esto se tradujo en que se tomó el mismo tipo de análisis que se realizó en el primer caso (UNAM), procurando conservar las mismas categorías, con la prerrogativa de encontrar resultados similares en el segundo caso (UCM) y con ello reforzar una propuesta teórica. En palabras de Yin (1989, p. 53): "Cada caso debe estar cuidadosamente seleccionado de manera que, o bien (a) prediga resultados similares (una replicación literal), o (b) produzca resultados contrarios, pero por razones predecibles (replicación teórica)"². En nuestra investigación entonces, la expectativa fue la de una replicación literal.

En el mismo tenor, Yin (1989) describe cuatro diferentes tipos de diseño para los estudios de caso. El nuestro correspondería al tipo 4, que consiste en casos múltiples (la UNAM y la UCM) dentro de los cuales se analizan a su vez múltiples unidades de análisis (36 y 31 profesores de posgrados de investigadores en educación, respectivamente).

Desde esta lógica de replicación, el estudio se llevó a cabo primeramente en la UNAM. Los datos se obtuvieron a través de la aplicación cara a cara de un instrumento en forma de cuestionario, que constaba de datos de identificación (sexo, edad y posgrado donde se colabora) y tres preguntas abiertas. Así, los participantes fueron seleccionados para un muestreo teórico en el cual se tuvieron en consideración tanto sus características personales como su disposición para participar con el estudio, respondiendo voluntariamente y de manera anónima a interrogantes acerca de las características de un(a) buen(a) profesor(a) universitario(a); sobre la manera en que se aprende a ser profesor(a) universitario(a); y, finalmente, recomendaciones para mejorar el trabajo académico en su universidad.

De acuerdo con Silverman (2010, p. 146), el muestreo teórico responde a criterios teóricos más que estadísticos, siendo que además cuenta con "mucha mayor flexibilidad que la mayoría de los diseños cuantitativos de investigación”. Así, se cuidó que fuera posible contar con sujetos de distinto sexo, edad y área de conocimiento. Por otra parte, el progresivo análisis, categorización y codificación de las respuestas de los participantes apuntó hacia una "saturación" de las categorías, término que se utiliza para referirse al momento en que las respuestas de los participantes dejan de aportar nuevos elementos para la construcción teórica (Strauss y Corbin, 2002). De acuerdo con estos autores:

La saturación consiste más bien en alcanzar el punto en la investigación, cuando la recolección de datos parece ser contraproducente porque lo "nuevo" que se descubre no le añade mucho a la explicación. $\mathrm{O}$, como sucede a veces, al investigador se le acaba el dinero o el tiempo o ambas cosas (Strauss y Corbin, 2002, p. 149). La técnica que se utilizó para el análisis consistió en la identificación de categorías conceptuales, para las cuales se codificaron las respuestas retomando las cinco competencias propuestas por Hirsch para la construcción de una escala que buscaba reflejar los rasgos más significativos de un buen profesional. Las categorías correspondientes a estas cinco competencias fueron la cognitiva, técnica, ética, social y afectivo-emocional (Hirsch, 2005). La elección se justifica en tanto que en esa investigación se interrogaba por las características del buen profesional a sujetos pertenecientes a diversos posgrados de investigación en educación

${ }^{2} \mathrm{El}$ original está en inglés y la traducción es nuestra. 
en comunidades educativas de España y México. En este sentido, nuevamente estamos considerando que el ejercicio de la docencia en este nivel educativo conlleva criterios de profesionalización, por lo que estas categorías teóricamente podrían aplicarse para describir la profesión docente, tanto como para cualquier otra profesión.

Las respuestas de los cuestionarios se transcribieron a una hoja de Excel, y en ella se llevó a cabo el proceso de codificación, basado en la propuesta de Coffey y Atkinson (2003), para quienes este consiste en,

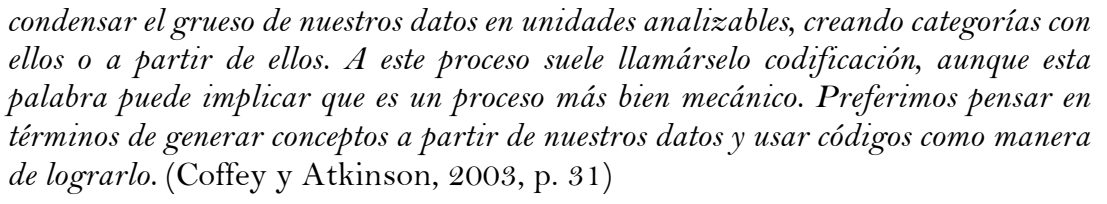

condensar el grueso de nuestros datos en unidades analizables, creando categorías con ellos o a partir de ellos. A este proceso suele llamárselo codificación, aunque esta palabra puede implicar que es un proceso más bien mecánico. Preferimos pensar en términos de generar conceptos a partir de nuestros datos y usar códigos como manera de lograrlo. (Coffey y Atkinson, 2003, p. 31)

Después de concluir con el análisis de resultados en la UNAM, fue llevado a cabo el mismo procedimiento en la UCM, replicando el estudio con 31 profesores, concluyendo con los levantamientos de información en noviembre de 2016. Los resultados de ambas instituciones de educación superior se muestran en el siguiente apartado.

\section{Resultados}

Para abordar los resultados de ambas instituciones, iremos realizando comparaciones retomando cada una de las tres preguntas abiertas que se plantearon a sus académicos de posgrado, comenzando con la que fue considerada como central para el desarrollo de este estudio, a saber, las características de un/a buen/a profesor/a de posgrado en la UNAM, o bien, en la UCM.

\subsection{Características de los buenos/buenas docentes de posgrado}

El primer factor de relevancia que cabe mencionar con respecto a la primera pregunta abierta del instrumento es que todas las respuestas, en ambas instituciones, se pudieron ajustar sin mayores inconvenientes a las cinco categorías de análisis que se retomaron a partir de Hirsch (2005): competencia cognitiva, técnica, ética, social y afectivo-emocional. Sin embargo, a partir del análisis de las diversas expresiones de los académicos de ambos posgrados, también se llevaron a cabo reformulaciones para describir de nueva cuenta cada una de estas cinco categorías. La cantidad de fragmentos analizados vinculados con cada categoría para las dos instituciones se muestra en el cuadro 1 y, como se puede ver, en general su estructura fue similar.

Cuadro 1. Fragmentos asociados con las categorías de análisis. Características de un/a buen/a profesor/a de posgrado en la UNAM y la UCM

\begin{tabular}{|c|c|c|}
\hline CATEGORÍAS & UNAM & UCM \\
\hline Cognitiva & 61 & 54 \\
\hline Técnica & 24 & 14 \\
\hline Ética & 21 & 18 \\
\hline Social & 23 & 21 \\
\hline Afectivo-emocional & 20 & 16 \\
\hline Total & 149 & 123 \\
\hline
\end{tabular}

Fuente: Elaboración propia. 
Por tratarse de un estudio de caso, a partir de una muestra reducida en cada uno de los dos escenarios, el margen de error o representatividad de estos porcentajes es desconocido, sin embargo, recordemos que uno de los propósitos de esta investigación fue caracterizar y comparar perfiles de los buenos docentes de posgrado de investigadores en educación en la UNAM y la UCM. Así, nuestros datos nos permiten inferir que entre los académicos de posgrado de estas universidades ocurren similitudes con respecto a la percepción que tienen en este sentido. También es posible identificar que eventualmente en ambas instituciones se llega a otorgar un papel predominante a los rasgos relacionados con la competencia cognitiva. Finalmente, se puede observar correspondencia en ambos escenarios con respecto a las cinco categorías o dimensiones de atributos para los buenos profesores de posgrado.

A continuación, nos enfocamos en la descripción de cada una de estas cinco categorías o dimensiones de análisis, con base en las aportaciones de los participantes en sendos estudios.

\subsubsection{Competencia cognitiva}

Esta categoría o dimensión agrupa los códigos que tienen como eje central el uso de facultades mentales racionales. Como cuando se menciona que el buen profesor de posgrado "debe estar al día sobre los conocimientos que debe impartir en su clase" (UCM 1-1) o si se hace referencia llanamente a su "dominio del tema" (UNAM 1-8).

Ahora bien, dentro de esta categoría principal de "Competencia cognitiva" el aspecto predominante en ambas instituciones -abarcando casi la mitad de los fragmentos discursivos identificados dentro de esta competencia- fue la adquisición, producción, posesión o difusión del conocimiento, lo que se codificó dentro de la subcategoría de "Conocimiento". Generalmente este conocimiento se refirió a la disciplina o asignatura en la que el docente colabora, sin embargo, también llegó a abarcar otros tipos de conocimientos, como sobre teorías pedagógicas, el contexto de los alumnos, o de carácter más general: "Amplios conocimientos no solo de la materia que imparte, sino de cultura en general" (UCM 1-11); "Conocimiento de metodologías recientes, disciplinares y didácticas" (UNAM 1-15).

En cualquier caso, el énfasis de las capacidades cognitivas se centró primordialmente en el conocimiento, pero esta no fue su única faceta. Algunas de las características deseables para la docencia en el posgrado también incluyeron aspectos como las capacidades para la investigación o para mantenerse actualizados. Estas dos fueron también subcategorías relevantes ubicadas dentro de la Competencia cognitiva, lo cual se observó en ambas instituciones: "Ser investigador para tener idea de cómo dirigir a los estudiantes a la investigación, o al menos de sus aplicaciones en el sector privado" (UNAM 1-17); "Pasión por la investigación” (UCM 1-6). "Afán de actualización y puesta al día en su disciplina" (UCM 1-9); "Actualización en los conceptos” (UNAM 1-9).

Otras subcategorías de la Competencia cognitiva también abarcaron aspectos como el pensamiento crítico-reflexivo, creatividad e innovación, o la disposición de una mente abierta para aprender. Sin embargo, su frecuencia de mención fue notablemente menor.

${ }^{3}$ Para las citas o referencias a las aportaciones de los entrevistados consideramos primeramente las siglas de la institución (UNAM o UCM), seguidas de la pregunta de referencia (1, 2 o 3) y finalmente el número del participante. 


\subsubsection{Competencia técnica}

En este rubro identificamos aquellas características que involucraron acciones concretas llevadas a cabo directamente por el docente, obedeciendo a una racionalidad instrumental, es decir, siguiendo un razonamiento lógico que persigue una modificación voluntaria y controlada de su entorno con una finalidad observable y específica.

Así, bajo esta dimensión destacaron en ambas instituciones dos subcategorías. Una de ellas correspondió a cuestiones pedagógicas y didácticas, como "Guiarles [a los estudiantes] para que aprendan a aprender" (UNAM 1-14), o el "dominio de las competencias docentes para organizar, estructurar, presentar la información y evaluarla” (UCM 1-28).

La otra subcategoría técnica preponderante se refirió concretamente a la capacidad de planeación, entendida esta como una habilidad para la gestión: "Ocasionalmente, implicación en la gestión académica" (UCM 1-27); "Planear y diseñar su actividad profesional, si es como profesor, si es como coordinador académico, como investigador.” (UNAM 1-28).

Cabe señalar que en esta categoría de Competencia técnica se esperaba una importante ocurrencia de menciones sobre la utilización de nuevas tecnologías aplicadas a los procesos educativos, así como sobre el uso de técnicas didácticas novedosas y centradas en el aprendizaje del alumno más que en la transmisión de información, dado que este es uno de los aspectos en los que las instituciones y la literatura sobre competencias de docentes universitarios suelen hacer más énfasis. Sin embargo, los comentarios al respecto fueron pocos y notoriamente mesurados, o hasta indirectos como lo reflejan estas menciones: "Adecuado uso de los recursos modernos para la docencia” (UNAM 1-12); o bien “adaptación a nuevas circunstancias” (UCM 1-19).

\subsubsection{Competencia ética}

Bajo esta dimensión agrupamos los códigos que hicieron referencia a criterios de valoración con los que se guían las acciones personales del docente. En este rubro se encontró una amplia gama de valores en ambas instituciones, con algunas coincidencias evidentes, pero también con expresiones que no dejaron muy en claro si los participantes podrían estarse refiriendo a lo mismo, o no. La dificultad para definir y evaluar este tipo de aspectos ha dado como resultado el que el factor ético sea pasado por alto en buena parte de la literatura sobre formación y evaluación de docentes universitarios. Sin embargo, coincidimos con Orellana, Merellano y Almonacid (2018) en señalar que la importancia de esta dimensión reclama su propio espacio, dadas las condiciones contextuales que retan a nuestras respectivas sociedades, ya que sin esta dimensión pierden sentido muchas de las actividades y actitudes implicadas en las otras.

La principal coincidencia en este sentido se dio a partir del "Compromiso", reflejado en ambas instituciones de manera explícita: "Compromiso con la universidad, la facultad, el departamento, los estudiantes” (UCM 1-8); “Que los alumnos sepan que eres una persona comprometida con tu trabajo...” (UNAM 1-1).

Otras expresiones en la UNAM apuntaron principalmente hacia la responsabilidad, en ocasiones utilizando otros términos, como "Ser cumplido" (UNAM 1-24), aunque también destacaron la humildad "para reconocer que no siempre se sabe todo" (UNAM 1-1) y el servicio a la sociedad, entre muchos otros. Por su parte, en la UCM también destacó el respeto: "Respeto hacia todo el alumnado y hacia sus ritmos y formas de aprender" (UCM 
1-22). Otras menciones también se refirieron a congruencia, honestidad o más ambiguamente a "valores" o a ser alguien ético en general.

\subsubsection{Competencia social}

En la dimensión de Competencia social se codificaron expresiones que resaltaban las capacidades del docente para interactuar con otros seres humanos; principalmente los estudiantes, aunque también otros docentes, o la sociedad en general.

En este ámbito destacó en primer lugar para ambas universidades la capacidad comunicativa, frecuentemente entendida como "Transmitir de forma clara los conocimientos" (UNAM 1-17), o más genéricamente como "Habilidades y actitudes comunicativas" (UCM 1-26). Eventualmente hubo coincidencia en manifestaciones de interés por los estudiantes, así como en las relaciones interpersonales con los mismos “incluso empatía, que no amistad” (UCM 1-4).

En este punto cabe aclarar que la razón por la que la competencia cognitiva está por separado de la competencia social y específicamente no incluye a las capacidades comunicativas, radica en que la posesión de conocimientos no necesariamente implica las habilidades para comunicarlos de manera didáctica. Así, podemos apreciar que, para los dos grupos de docentes interrogados, en general la posesión del conocimiento tenía mayor relevancia que el contar con habilidades especiales para poder comunicarlo.

\subsubsection{Competencia afectivo-emocional}

Como dimensión o categoría, la Competencia afectivo-emocional abarcó dos subcategorías. Una hizo referencia a la capacidad emocional, lo que incluyó cuestiones relacionadas con empatía, entusiasmo o motivación, entre otras. "Empatía con los alumnos, pues alguna vez estuviste en ese rol” (UNAM 1-1); “implicación, motivación, interés por la educación” (UCM 1-26) y “tolerancia a la frustración” (UCM 1-26) son algunos ejemplos de manifestaciones de esta subcategoría.

La segunda parte de la Competencia afectivo-emocional correspondió a la "Identificación con la profesión”, que incluyó emociones como el "gusto por el trabajo" (UNAM 1-13); "debe gustarle impartir docencia” (UCM 1-1), o también la llamada "Vocación a su carrera" (UNAM 1-28) en tanto que esta se vincula con emocionalidad profunda en relación con la actividad profesional: "Vocación, Pasión por la investigación” (UCM 1-6).

Es interesante que este fue un aspecto en el que las tendencias difirieron entre la UNAM y la UCM, pues mientras en la primera se enfatizó más en las capacidades emocionales, en la segunda se resaltó más la identificación con la profesión. Sin embargo, el tamaño de las muestras impide realizar inferencias al respecto, y solo cabría sugerir realizar más investigación sobre este aspecto a fin de verificar si en ello existen diferencias significativas.

\section{2. ¿Cómo se llega a ser un buen profesor de posgrado?}

Las respuestas a esta pregunta también revelaron semejanzas interesantes en la percepción de los académicos de posgrado de la UNAM y la UCM, con respecto a lo que consideran importante para llegar a ser buenos profesores. De sus respuestas se pueden rescatar orientaciones para las estrategias de formación docente.

Para el análisis de las respuestas se eligió continuar con las mismas categorías o dimensiones que se utilizaron en la pregunta anterior, debido principalmente a que entre 
ambas podría haber una estrecha relación. Así, encontramos que la mayoría de las respuestas en ambas instituciones se concentraron en el ámbito de la Competencia cognitiva. En este sentido se identificaron comentarios como: "a través de estudios, lecturas, conversaciones, observaciones e investigaciones propias y ajenas" (UCM 2-30) o "Tomando clases de posgrado. Estudiando varias fuentes bibliográficas. Teniendo recursos extras de actualización metodológica y disciplinar" (UNAM 2-19).

Asimismo, en las dos instituciones, lo más mencionado dentro de esta subcategoría predominante fue el conocimiento obtenido a partir de la experiencia, lo cual, a pesar de estar estrechamente vinculado con una actividad práctica, requiere previamente asumirse en un proceso de aprendizaje. Esto se pudo ver reflejado en comentarios como "No hay recetas, se aprende (o no) sobre la marcha" (UNAM 2-9) o bien:

\begin{abstract}
Principalmente, aprendiendo desde la propia práctica docente. A menudo este aprendizaje implica probar cosas nuevas y ver cómo funcionan en el aula, ir probando distintas metodologías y herramientas para ver cómo funcionan e ir reflexionando sobre la propia actividad docente (¿̨ha funcionado, he conseguido los objetivos que tenía, han aprendido los estudiantes, se han motivado...?) (UCM 2-22).
\end{abstract}

Otra forma de aprendizaje que aparece con cierta frecuencia consiste en tomar a otros docentes como un ejemplo, ya sea como modelo a seguir o para no cometer sus mismos errores: "analizando los errores y aciertos de mis profesores del pasado" (UCM 2-10); "Al tener clase con profesores comprometidos y talentosos que motivan el buen ejercicio de la profesión” (UNAM 2-21).

Aparte de la dimensión correspondiente a la Competencia cognitiva, las otras categorías fueron de escasa o nula utilidad. En ocasiones se pudieron observar algunos fragmentos apelando a la dimensión de Competencia social, especialmente aludiendo a la subcategoría comunicativa al contemplar la obtención de retroalimentación por parte de pares y alumnado: "Por el intercambio de ideas con colegas y, sobre todo con estudiantes; si estos no pueden aprender como yo enseño, entonces intento enseñar como ellos aprenden." (UNAM 2-10). Eventualmente también se mencionó el apoyo en herramientas técnicas como la evaluación: "a través de encuestas propias y DOCENTIA"" (UCM 2-2); "Sometiendo mi trabajo a evaluación para mejorar y evitar errores" (UCM 2-9). En cuanto a las dimensiones ética y afectivo-emocional se usaron poco, y en el caso de la UCM para esta pregunta, no se utilizaron en absoluto.

\title{
3.3. Sobre las recomendaciones para mejorar el trabajo académico
}

La tercera pregunta abierta del instrumento que utilizamos para recabar percepciones de los académicos de posgrado en la UNAM y la UCM, solicitaba recomendaciones a los mismos para mejorar el trabajo académico. Al igual que en las dos preguntas anteriores, se utilizaron las mismas cinco categorías o dimensiones para analizar las respuestas, que nuevamente fueron funcionales para codificarlas todas, permitiendo en cierta manera comparar diversos tipos de expresiones entre tres preguntas diferentes. Esta vez, sin embargo, se encontraron notorias desigualdades entre los comentarios de los participantes.

A diferencia de las dos primeras preguntas, donde predominó la dimensión cognitiva, en la tercera pregunta prevaleció para ambas instituciones la categoría social, aunque por

${ }^{4}$ DOCENTIA es un programa interinstitucional para la evaluación del profesorado universitario español. 
diferentes motivos. En este sentido, mientras que para los académicos de la UNAM la mayoría de las recomendaciones redundaron sobre aspectos de mejora en la comunicación con los estudiantes, en la UCM sobresalieron las sugerencias de mejoras implementadas desde ámbitos institucionales, impactando de alguna manera en las condiciones de trabajo.

Así, en la UNAM se recuperaron expresiones como "Reuniones de retroalimentación" (UNAM 3-11), "Enfocarse en transmitir los fundamentos (los conocimientos fundamentales) y no todos los detalles de ellos" (UNAM 3-17) o "Saber escuchar y tratar de entender y si es necesario, estudiar sus propuestas [de los estudiantes] para tratar de entenderlas" (UNAM 3-6). En cambio, en la UCM encontramos expresiones que se asemejaron más a una petición: "también podría ser una buena idea fomentar (como se hace en países anglosajones) las redes informales de profesorado en cada área de conocimiento para ayudar a las nuevas generaciones a incorporarse a la docencia” (UCM 3-29); "algo que falta es un servicio de apoyo a la edición y traducción de artículos" (UCM 3-18), o "Mayor dedicación a estas tareas y también mejores salarios. Con un profesorado en precario no se puede realizar un buen trabajo académico, ni formar equipos de investigación que impulsen el desarrollo del conocimiento.” (UCM 3-24).

Al observar otras recomendaciones que siguieron en frecuencia de menciones a las de la dimensión comunicativa, en la UNAM encontramos la necesidad de reforzar el compromiso del profesorado, bajo distintas formulaciones: “dedicación y pasión por enseñar” (UNAM 331), "Tener determinación de hacer bien las cosas" (UNAM 3-7); o "Dejar de interesarse solo en puntos del SNI (Sistema Nacional de Investigadores) y comprometerse a generar discusiones académicas y sociales” (UNAM 3-4). Todo ello atañe a la dimensión ética, en tanto que apela a valores que tendrían que asumirse personalmente para guiar cambios en el comportamiento de los sujetos.

En contraste con esto, para la UCM las recomendaciones de carácter institucional estuvieron seguidas de otras concernientes al conocimiento especializado: "Repensar los contenidos y las competencias en función de la materia que impartimos y de las posibilidades futuras de profesionalización de los estudiantes" (UCM 3-13); "Conocer mejor qué demanda la sociedad para dar la materia enfocada a esas necesidades” (UCM 3-3); o "Establecer planes de formación docente para los profesores de nuevo ingreso y de reciclaje para los que llevamos tiempo en la docencia” (UCM 3-28).

Otras sugerencias en la UNAM apuntaron también hacia el alumnado, como "Poner atención a las posibles dificultades de los estudiantes y buscar la manera de superar sus deficiencias y aprovechar sus fortalezas" (UNAM 3-10), o "Incluir más ejemplos para facilitar la comprensión” (UNAM 3-3). Por su parte, en la UCM también se hicieron menciones sobre el ámbito pedagógico-didáctico, como "Programar cursos específicos de pedagogía adecuada a las características del estudiante universitario y a los objetivos de cada grado o postgrado" (UCM 3-13) y a la colaboración con pares: "Consultar con otros compañeros sus métodos y experiencias docentes" (UCM 3-6); "Que hubiese más comunicación entre los propios compañeros a fin de conocer más las aportaciones que cada uno hace o el grupo de investigación" (UCM 3-20); o "Mayor coordinación entre el profesorado para no repetir contenidos, organizar la carga de trabajo de los estudiantes, etc..." (UCM 3-22). Este último aspecto también pudo identificarse en algunos comentarios en la UNAM: “Tener intercambio y contacto con colegas de otras disciplinas y países" (UNAM 3-20); "Como docente considero que el intercambio con otros docentes permite 
aprender y mejorar nuestra práctica. Aprender cómo otros dan salida a las dificultades que nos presenta la docencia y si no para reorientar mi propia práctica” (UNAM 3-24).

Finalmente, sobresalió una ausencia poco esperada, tanto en la UNAM como en la UCM, y fue nuevamente con respecto al uso de las nuevas tecnologías. Al igual que en la primer pregunta, se mencionaron poco y, cuando aparecieron, estuvieron rodeadas de matices y cautelas que iban más allá del simple dominio y actualización tecnológica: "Sabemos usar las nuevas tecnologías, tenemos competencias digitales, más o menos avanzadas, pero no sabemos necesariamente como construir el conocimiento con el alumnado en el nuevo entorno digital y en relación con las «nuevas» metodologías" (UCM 3-29); "Evitar usar o abusar de presentaciones Power Point” (UNAM 3-19).

Como se puede observar, en esta tercera pregunta hubo algunas similitudes significativas, aunque también encontramos en ella las mayores diferencias. Atribuimos esto al contexto de ambas universidades, lo que sugiere que, a pesar de contar con finalidades similares, las circunstancias de cada una reclaman estrategias de mejora con características particulares.

\section{Conclusiones}

El objetivo de este estudio era indagar las principales características del buen docente universitario de posgrados de investigadores en educación para poder construir una perspectiva de la calidad de la formación que imparten. El motivo era doble: por un lado, la escasez de estudios específicos sobre los docentes de posgrados y, por otro, la falta de estudios sobre la muestra utilizada (posgrados de investigadores en educación).

Si bien los estudios sobre el buen docente de posgrado son escasos, la literatura sobre las características del buen profesorado universitario es profusa. Esto hace posible dilucidar algunas tendencias generales, si bien no hay consensos sobre cuáles deberían de ser estas características, mientras que las metodologías para su análisis y valoración son muy diversas. Esta situación podría deberse a las demandas del contexto en cada institución, y por ello el estudio múltiple de casos puede contribuir en este sentido.

Al comparar las percepciones sobre lo que debería ser el perfil ideal del buen profesor universitario, en este caso aplicado a profesorado de títulos de posgrado de investigadores en educación, lo que encontramos en esta investigación nos indica que entre la UNAM y la UCM ocurren notorias coincidencias desde la percepción de los académicos de posgrado, pero también algunas diferencias relevantes. Así, por ejemplo, el predominio de la Competencia cognitiva o las pocas menciones sobre el manejo de tecnologías fueron una constante en ambas instituciones, mientras que la necesidad de más apoyo por parte de la universidad solo destacó en una de ellas.

Las otras categorías, correspondientes a las competencias técnicas, sociales, éticas y afectivo-emocionales son relevantes en conjunto, pero al interior de ellas existe una gran diversidad de características que, al ser comparadas con la Competencia cognitiva, pierden peso estratégico. Este es un dato significativo, en tanto que nos muestra un colectivo docente enfocado en la posesión de datos especializados y actualizados (Competencia cognitiva), mientras que los nuevos paradigmas educativos insisten en enfocarse en la resolución de problemas para lo que no se puede prescindir de una perspectiva social (Wee y Monarca, 2019). Al mismo tiempo, la información específica se encuentra cada vez más en espacios virtuales al alcance de usuarios en todo el mundo. Todo ello apunta hacia una necesidad apremiante de replantear la educación -como se ha señalado ya en estancias 
internacionales (UNESCO, 2015)- no solo en los niveles de primaria y secundaria, sino en el superior y particularmente también en el posgrado.

Asimismo, es notorio que los procesos formativos del profesorado en este nivel, más allá de cursos y lecturas especializadas, se constituyen principalmente a través de adquirir experiencia práctica como docentes, así como en el intercambio de dichas experiencias con otros colegas del posgrado. En cuanto a las sugerencias para mejorar el trabajo académico, para la UNAM sobresale la necesidad de reforzar aspectos comunicativos, principalmente a través de la capacidad de transmisión del profesorado, mientras que en la UCM predominaron las peticiones de mejores condiciones de apoyo por parte institucional, tanto en cuestiones formativas como laborales.

Por otra parte, la buena docencia universitaria se ha abordado mayormente hasta ahora en la literatura sin especificar características, necesidades o tendencias concretas del posgrado. Algunas tendencias sobre la calidad docente universitaria en los últimos años han puesto el acento en la necesidad de competencias relacionadas con la innovación, el uso de las TIC o las habilidades comunicativas y didácticas (Cabero, 2014; Hoyuelos e Ibáñez, 2018; Martín y Montilla, 2016; Salinas, 2004), pero lo encontrado en nuestro estudio indica una perspectiva centrada en la Competencia cognitiva y, concretamente, en la formación permanente disciplinar, favoreciendo el conocimiento profundo de la materia así como una cultura más general. Sorprendentemente, esto no se contempla o valora directamente por ninguna de las instituciones que certifican la calidad de la docencia en México o España, siendo que estas características del buen docente universitario también aparecen en otras investigaciones ya mencionadas, como la de Fernández-Cruz y Romero (2010) o Monereo y Domínguez (2014).

La importancia dada a esta cuestión también hace plantear la necesidad de un mayor apoyo institucional al docente universitario para que tenga tiempo y recursos a su disposición para poder actualizarse y profundizar en sus líneas de trabajo (una cuestión en la que la UNAM respalda notablemente a sus investigadores, que son quienes constituyen la gran mayoría de sus profesores de posgrado). El acceso a las bases de datos más prestigiosas, el apoyo económico para la asistencia a reuniones internacionales (jornadas, seminarios y congresos, entre otros) y para la estancia en otras instituciones de educación superior o centros de investigación, así como las ayudas a la investigación a través de suficientes y adecuadas convocatorias son solo algunos de los requisitos para una buena docencia en el nivel de posgrado.

Las diferencias contextuales sugieren que los parámetros para la evaluación de una buena docencia se establezcan de manera particular en cada caso pues, por una parte, existe una gran diversidad de indicadores o criterios asociados con ella, con diferentes ponderaciones encontradas en la literatura sobre el tema. Por otra parte, las circunstancias de cada institución, e incluso sus respectivos entornos nacionales, pueden influir sobre la percepción de las necesidades del profesorado y sus alternativas formativas.

Atendiendo al perfil docente identificado, podríamos considerar que se tiende a un modelo más centrado en la técnica de transmisión, en el que las competencias cognitivas, técnicas y sociales adquieren un mayor protagonismo. No obstante, como sostenía Reyero (2014), este modelo es compatible con el que prima la relación que genera la transmisión, conformado por las competencias éticas y afectivo-emocionales. La práctica de la docencia de posgrado no solo tiene consecuencias en el prestigio de las instituciones sino también en la futura vida profesional de sus estudiantes lo que, consecuentemente, tendrá un gran 
impacto en la sociedad. Los altos niveles de competitividad presentes hoy día en los distintos ámbitos laborales a los que se enfrentan los investigadores en educación precisan una formación exigente y, para ello, los docentes de posgrado que forman a los futuros profesionales deben asimismo tener una formación que responda a estos requerimientos.

La trascendencia de la calidad de la docencia de posgrado hace necesario, por todo ello, replantear y fortalecer los procesos de selección, formación y promoción de sus docentes y para esto se hacen necesarias investigaciones que identifiquen las necesidades y las limitaciones actuales, así como aquellas fortalezas que deben ser promovidas y aquellos recursos que puedan hacer posible un alto nivel de calidad. Con este trabajo se ha ofrecido un acercamiento a la realidad académica de este colectivo a través de los propios docentes de posgrado de investigadores en educación de dos de las más importantes instituciones de educación superior de Iberoamérica, lo que supone una aportación original por cuanto la literatura sobre este colectivo es prácticamente inexistente.

Las limitaciones del presente trabajo están relacionadas, en primer lugar, con la falta de estudios previos de investigación específica sobre los docentes de posgrados de investigadores en educación por lo que, para sentar las bases para entender el objeto de esta investigación, se ha tenido que acudir a investigaciones más generales sobre caracterización del buen docente universitario. En segundo lugar, los estudios de caso no cuentan con una representatividad conocida. Sus aportaciones no permiten saber qué porcentaje de una población opina de cierta manera, o cuántos se encuentran bajo ciertas condiciones. Aun así, su reconocimiento está en aumento, en tanto permiten describir situaciones que efectivamente están ocurriendo y que, de repetirse ciertas circunstancias, las características observadas también se pueden repetir. Así, para el caso del presente estudio no podemos saber cuántos profesores de posgrado piensan de cierta forma, o si en general la mayoría percibe cierta característica como un elemento primordial de la docencia de excelencia. Sin embargo, sí ha sido posible identificar que en dos escenarios distintos ocurren similitudes y que en ambos se encuentran colectivos de docentes identificando cualidades específicas en profesores de excelencia.

También ha sido posible identificar que el modelo de categorías es útil para describir los hallazgos en ambos escenarios. Con esta información se cuenta también con indicios de lo que podría estar aconteciendo con la población general de docentes de posgrado en ambos países -y que probablemente también se podría llegar a encontrar en otros escenarios, incluso en otros países- pero, sobre todo, se cuenta con un modelo con muchas posibilidades para ser replicado en poco tiempo y con recursos moderados, aún en escenarios muy distintos o distantes. De ahí una de las aportaciones metodológicas de este estudio para la investigación y mejor comprensión de la buena docencia en cualquier escenario de posgrado.

\section{Referencias}

Arbesú, M., Canales, A., Crispín, M., Cruz, I., Figueroa, A. y Gilio, M. (2011). Las políticas y los usos de la evaluación de la docencia en la educación superior: Planteamientos y perspectivas. En M. Rueda y F. Díaz-Barriga (Coords.), La evaluación de la docencia en la universidad. Perspectivas desde la investigación y la intervención profesional, (pp. 203-257). Ciudad de México: Plaza y Valdéz.

Bausela, E. (2004). Formación inicial del profesorado universitario. Campo abierto: Revista de Educación, 25, 63-74. 
Belando-Montoro, M. R. y Tavárez, M. (2017). El perfil del docente en la educación universitaria de República Dominicana. International Journal of Educational Research and Innovation, 7, 164185 .

Cabero, J. (2014). Formación del profesorado universitario en TIC. Aplicación del método Delphi para la selección de los contenidos formativos. Educación XX1, 17(1), 111-132. https://doi.org/10.5944/educxx 1.17.1.10707

Carlos, J. (2011). La calidad de la enseñanza en educación superior ¿Qué es una buena enseñanza en este nivel educativo? Perfiles Educativos, 33(núm. especial), 129-141.

Casero, A. (2010). ¿Cómo es el buen profesor universitario según el alumnado? Revista Española de Pedagogía, 246, 223-242.

Coffey, A. y Atkinson, P. (2003). Encontrar sentido a los datos cualitativos. Estrategias complementarias de investigación. Bogotá: Universidad de Antioquía.

Fernández, A. (2008). La formación inicial del profesorado universitario: El título de especialista universitario en pedagogía universitaria de la Universidad Politécnica de Valencia. Revista Interuniversitaria de Formación del Profesorado, 63(3), 161-187.

Fernández, N. (2007). La evaluación y la acreditación de la calidad: Situación, tendencias y perspectivas. En IESALC (Coord.), Informe sobre la educación superior en América Latina y el Caribe 2000-2005. La metamorfosis de la educación superior (pp. 33-42). Caracas: UNESCO.

Fernández-Cruz, M. y Romero, A. (2010). Indicadores de excelencia docente en la Universidad de Granada. Revista Portuguesa de Pedagogía, 44(1), 83-1 17. https://doi.org/10.14195/16478614_44-1_4

García, J. y Medécigo, A. (2014). Los criterios que emplean los estudiantes universitarios para evaluar la in-eficacia docente de sus profesores. Perfiles Educativos, 34(143), 124-139. https://doi.org/10.1016/So185-2698(14)70613-0

García-Gallego, A., Georgantzis, N., Martín-Montaner, J. y Pérez-Amaral, T. (2015). La calidad percibida de la docencia universitaria: ¿Son mejores docentes los mejores investigadores? $e$ pública: Revista Electrónica sobre la Enseñanza de la Economía Pública, 16, 49-66.

Gargallo, B., Sánchez, F., Ros, C. y Ferreras, A. (2010). Estilos docentes de los profesores universitarios. La percepción de los alumnos de los buenos profesores. Revista Iberoamericana de Educación, 51(4), 37-61.

Guzmán, J. C. (2018). Las buenas prácticas de enseñanza de los profesores de educación superior. REICE. Revista Iberoamericana sobre Calidad, Eficacia y Cambio en Educación, 16(2), 133-149. https://doi.org/10.15366/reice2018.16.2.008

Hamer, A. (2015). La percepción del buen profesor en alumnos de nuevo ingreso a la enseñanza universitaria: El caso de ETEA (Córdoba). Revista Complutense de Educación, 26(2), 227-240. https://doi.org/10.5209/rev_RCED.2015.v26.n2.41534

Hirsch, A. (2005). Construcción de una escala de actitudes sobre ética profesional. Revista Electrónica de Investigación Educativa, 7(1), 27-45.

Hortigüela, D., Ausín, V., Delgado, V. y Abella, V. (2017). Análisis de la importancia de los criterios de evaluación y el reconocimiento académico docente universitario como indicadores de la calidad educativa en España. Revista de la Educación Superior, 46(181), 75-87. https://doi.org/10.1016/j.resu.2016.10.002

Hoyuelos, F. y Ibáñez, J. (2018). Características y prácticas docentes universitarias más valoradas por los/as estudiantes de primer curso comparadas en los grados en química y ciencia y tecnología de los alimentos de la Universidad de Burgos. Revista Complutense de Educación, 29(2), 423-439. https://doi.org/10.5209/RCED.52784 
Luna, E., Valle, M. y Osuna, C. (2010). Los rasgos de un buen profesional, según la opinión de estudiantes universitarios en México. Revista Electrónica de Investigación Educativa, 12, 1-14.

Martín, A. y Montilla, M. (2016). El uso del blog como herramienta de innovación y mejora de la docencia universitaria. Profesorado. Revista de Currículum y Formación del Profesorado, 20(3), 659-686.

Martínez, E. (2010). Ética profesional de los profesores. Bilbao: Desclée de Brouwer.

Matus, J. y Linares, C. (2016). La carrera docente y la profesionalización de la docencia. Reencuentro, 71, 85-106.

McAleese, M., Bladh, A., Berger, V., Bode, C., Muehlfeit, J., Petrin, T., Schiesaro, A. y Tsoukalis, L. (2013). Report to the European commission on improving the quality of teaching and learning in Europe's higher education institutions. Luxemburgo: Publications Office of the European Union.

Miguel, M. de (2003). Calidad de la enseñanza universitaria y desarrollo profesional del profesorado. Revista de Educación, 331, 13-34.

Monereo, C. y Domínguez, C. (2014). La identidad docente de los profesores universitarios competentes. Educación XX1, 17(2), 83-104. https://doi.org/10.5944/educxx 1.17.2.11480

Moreno, T. (2018). La evaluación docente en la universidad: Visiones de los alumnos. REICE. Revista Iberoamericana sobre Calidad, Eficacia y Cambio en Educación, 16(3), 87-101. https://doi.org/10.15366/reice2018.16.3.005

Orellana, R., Merellano, E. y Almonacid, A. (2018). Buen o buena docente de universidad: Perspectiva del personal directivo de carrera y de los mismos grupos docentes. Revista Electrónica Educare, 22(2), 88-114. https://doi.org/10.15359/ree.22-2.6

Patiño, H. (2012). Educación humanista en la universidad. Un análisis a partir de las prácticas docentes efectivas. Perfiles Educativos, 34(136), 23-41.

Patiño, H. (2015). ¿Qué hacen los docentes de excelencia? Claves para la formación humanista en la universidad. Ciudad de México: Universidad Iberoamericana.

Reyero, D. (2014). La excelencia docente universitaria. Análisis y propuestas para una mejor evaluación del profesorado universitario. Educación XX1, 17(2), 125-143. https://doi.org/10.5944/educxx 1.17.2.11482

Rocha, R. (2012). La docencia universitaria desde la perspectiva de los alumnos frente a la de los profesores. Innovación Educativa, 12(58), 91-118.

Rocha, R. (2013). Escala de opinión de los estudiantes sobre la efectividad de la docencia en educación superior. Formación Universitaria, 6(6), 13-22. https://doi.org/10.4067/So7 18-50062013000600003

Ruiz-Corbella, M. y Aguilar-Feijoo, R. (2017). Competencias del profesor universitario: Elaboración y validación de un cuestionario de autoevaluación. Revista Iberoamericana de Educación Superior, 8(21), 37-65.

Salinas, J. (2004). Innovación docente y uso de las TIC en la enseñanza universitaria. Revista Universidad y Sociedad del Conocimiento, 1(1), 23-45.

Silverman, D. (2010). Doing qualitative research. Londres: Sage.

Strauss, A. y Corbin, J. (2002). Bases de la investigación cualitativa. Técnicas y procedimientos para desarrollar la teoría fundamentada. Medellín: Universidad de Antioquía.

Torra, I., Corral, I., Pérez, M., Triadó, X., Pagés, T., Valderrama, E., ... y Tena, A. (2012). Identificación de competencias docentes que orienten el desarrollo de planes de formación 
dirigidos a profesorado universitario. Revista de Docencia Universitaria, 10(2), 21-56.

https://doi.org/10.4995/redu.2012.6096

UNESCO. (2015). Replantear la educación. ¿̇Hacia un bien común mundial? París: UNESCO.

Vera, H. (2017). El homo academicus y la máquina de sumar: Profesores universitarios y la evaluación cuantitativa del mérito académico. Perfiles Educativos, 39(155), 87-106.

https://doi.org/10.22201/iisue.24486167e.2017.155.58107

Villa, A. (2008). La excelencia docente. Revista de Educación, 346, 177-212.

Wee, C. y Monarca, H. (2019). Educación superior en contextos de cuasi-mercados. Educación XX1, 22(1), 117-138. https://doi.org/10.5944/educxx 1.20047

Yin, R. (1989). Case study research. Newbury Park: Sage.

Zabalza, M. A. (2009). Ser profesor universitario hoy. La Cuestión Universitaria, 5, 69-81.

\section{Breve CV de los autores}

\section{María R. Belando-Montoro}

Doctora en Pedagogía. Profesora Titular del Departamento de Estudios Educativos en la Facultad de Educación de la Universidad Complutense de Madrid. Ha sido coordinadora del Máster en Estudios Avanzados en Educación Social y en la actualidad es Vicedecana de Relaciones Internacionales. Ha dirigido varios proyectos de innovación docente sobre calidad en la enseñanza universitaria. Sus líneas de investigación están relacionadas con: calidad y excelencia del profesorado universitario, formación continua, aprendizajeservicio y gerontología educativa. Sobre dichas líneas ha trabajado en proyectos de investigación nacionales, europeos e iberoamericanos y tiene publicaciones nacionales e internacionales de impacto. Es evaluadora-experto independiente del Programa Erasmus+ y de la Research Executive Agency de la Comisión Europea. ORCID ID: http://orcid.org/oooo-0001-5730-7896. Email: mbelando@ucm.es

\section{José Francisco Alanís Jiménez}

Licenciado en Ciencias de la Comunicación (UIC/CDMX), Experto en servicios de información juvenil e información al ciudadano (USAL/España), Maestría en Desarrollo Huma no (UIA/Puebla), Doctorado en Educación (UAEM/Morelos), Posdoctorado en el Posgrado de Pedagogía 2015-2017 (UNAM/CDMX). Candidato del Sistema Nacional de Investigadores (2018-2020). UNAM, becario del programa de becas posdoctorales en la UNAM, Centro Regional de Investigaciones Multidisciplinarias, Asesorado por la Dra. Carlota Guzmán Gómez. Trabajo en temas relacionados con educación superior. ORCID ID: http://orcid.org/OOOO-0002-3092-3397.Email: picoalanis@gmail.com 